\title{
The Structure and Function of Ionotropic Receptors in Drosophila
}

\author{
Lina $\mathrm{Ni}^{*}$ \\ School of Neuroscience, Virginia Tech, Blacksburg, VA, United States
}

lonotropic receptors (IRs) are a highly divergent subfamily of ionotropic glutamate receptors (iGluR) and are conserved across Protostomia, a major branch of the animal kingdom that encompasses both Ecdysozoa and Lophothrochozoa. They are broadly expressed in peripheral sensory systems, concentrated in sensory dendrites, and function in chemosensation, thermosensation, and hygrosensation. As iGluRs, four IR subunits form a functional ion channel to detect environmental stimuli. Most IR receptors comprise individual stimulus-specific tuning receptors and one or two broadly expressed coreceptors. This review summarizes the discoveries of the structure of IR complexes and the expression and function of each IR, as well as discusses the future direction for IR studies.

Keywords: Drosophila, ionotropic receptors, chemosensation, gustation, olfaction, thermosensation, hygrosensation

\section{INTRODUCTION}

Ionotropic receptors (IRs) are a highly divergent subfamily of ionotropic glutamate receptors (iGluR) (Benton et al., 2009). Most iGluRs bind the excitatory neurotransmitter glutamate and function in synaptic communication in the brain (Mayer and Armstrong, 2004). In contrast, IRs are primarily and broadly expressed in peripheral sensory systems and have diverse functions,

OPEN ACCESS

Edited by:

Masahito Yamagata, Harvard University, United States

Reviewed by:

Youngseok Lee, Dankook University, South Korea

Rui Xiao,

University of Florida, United States

${ }^{*}$ Correspondence:

Lina $\mathrm{Ni}$

linani@vt.edu

Received: 07 December 2020 Accepted: 28 December 2020 Published: 01 February 2021

Citation:

Ni L (2021) The Structure and Function of lonotropic Receptors in

Drosophila.

Front. Mol. Neurosci. 13:638839. doi: 10.3389/fnmol.2020.638839 including chemosensation, thermosensation, and hygrosensation (van Giesen and Garrity, 2017). They might also be involved in hearing and social cluster formation (Senthilan et al., 2012; Jiang et al., 2020). IRs are conserved across Protostomia, a major branch of the animal kingdom that encompasses both Ecdysozoa and Lophothrochozoa, and play a key role in host-seeking behavior in disease vectors, such as mosquitoes (Croset et al., 2010; Raji et al., 2019; Greppi et al., 2020; Jové et al., 2020). While "antennal" IRs are conserved across insects, often contain many introns, and function in olfaction, thermosensation, and hygrosensation; species-specific "divergent IRs" are often single intron genes, are expressed in peripheral and internal gustatory neurons, and are required for taste and food assessment (Benton et al., 2009; Croset et al., 2010).

As iGluRs, four IR subunits form a functional ion channel to allow cations-mainly monovalent cation, but also calcium-to flux into and activate sensory neurons (Abuin et al., 2011, 2019). Most IR receptors comprise individual stimulus-specific tuning receptors and one or two broadly expressed coreceptors (Abuin et al., 2011). IR proteins are detected in the cell body and sensory dendrites but not at synapses (Benton et al., 2009; Ai et al., 2010, 2013; Abuin et al., 2011; Grosjean et al., 2011). Expression of IRs in sensory dendrites is critical for sensory responses and requires the heteromeric IR complex formation (Abuin et al., 2011; Ai et al., 2013).

There are 63 IR proteins in Drosophila melanogaster, including four coreceptors (IR8a, IR25a, IR76b, and IR93a) and 59 tuning receptors (Benton et al., 2009; Koh et al., 2014; van Giesen and Garrity, 2017). The expression of most IRs-except for five tuning receptors (IR48a, IR51a, IR54a, IR60a, and IR87a)-has been described (Supplementary Tables 1, 2). However, the function of only 18 tuning receptors has been found (Table 1). 
In this review, I will first summarize the discoveries of the structure of IR complexes. Next, I will discuss the known function of IR proteins, including the discoveries from most recent studies as well as the information described in previous review articles (Rytz et al., 2013; van Giesen and Garrity, 2017; Rimal and Lee, 2018). Importantly, the information about IR expression and function, with references, will be listed in Table 1 and Supplementary Tables 1, 2. Finally, I will discuss the future direction for IR studies in Drosophila melanogaster and other animals.

\section{STRUCTURE}

As iGluRs, four IR subunits are predicted to form a functional ion channel. This possibility has been confirmed by photobleaching and counting GFP-tagged subunits in vitro and analogy with the heterotetrameric stoichiometry of iGluRs (Abuin et al., 2011,
2019). For IR complexes that use IR8a as coreceptors, there are two IR8a subunits and two tuning IRs in each heterotetrameric complex (Abuin et al., 2011, 2019). Although IRs are iGluRrelated proteins, they are not closely related to the well-described AMPA, NMDA, or kainate classes of iGluRs (Benton et al., 2009). Instead, the IR family is extremely divergent and the overall amino acid sequence identity is $10-70 \%$ (Benton et al., 2009).

iGluRs contain four protein domains: the amino-terminal domain (ATD), ligand-binding domain (LBD), transmembrane domain (TMD), and carboxy-terminal domain (CTD) (Mayer and Armstrong, 2004). The extracellular ATD domain is followed by the LBD domain that contains two half-domains, S1 and S2. S1 and S2 form a "Venus flytrap" structure that closes upon binding of glutamate (Armstrong et al., 1998). In the primary structure, S1 and S2 are separated by the ion channel pore. The ion channel pore is formed by two transmembrane segments (TM1 and TM2)

TABLE 1 | The known function of each IR.

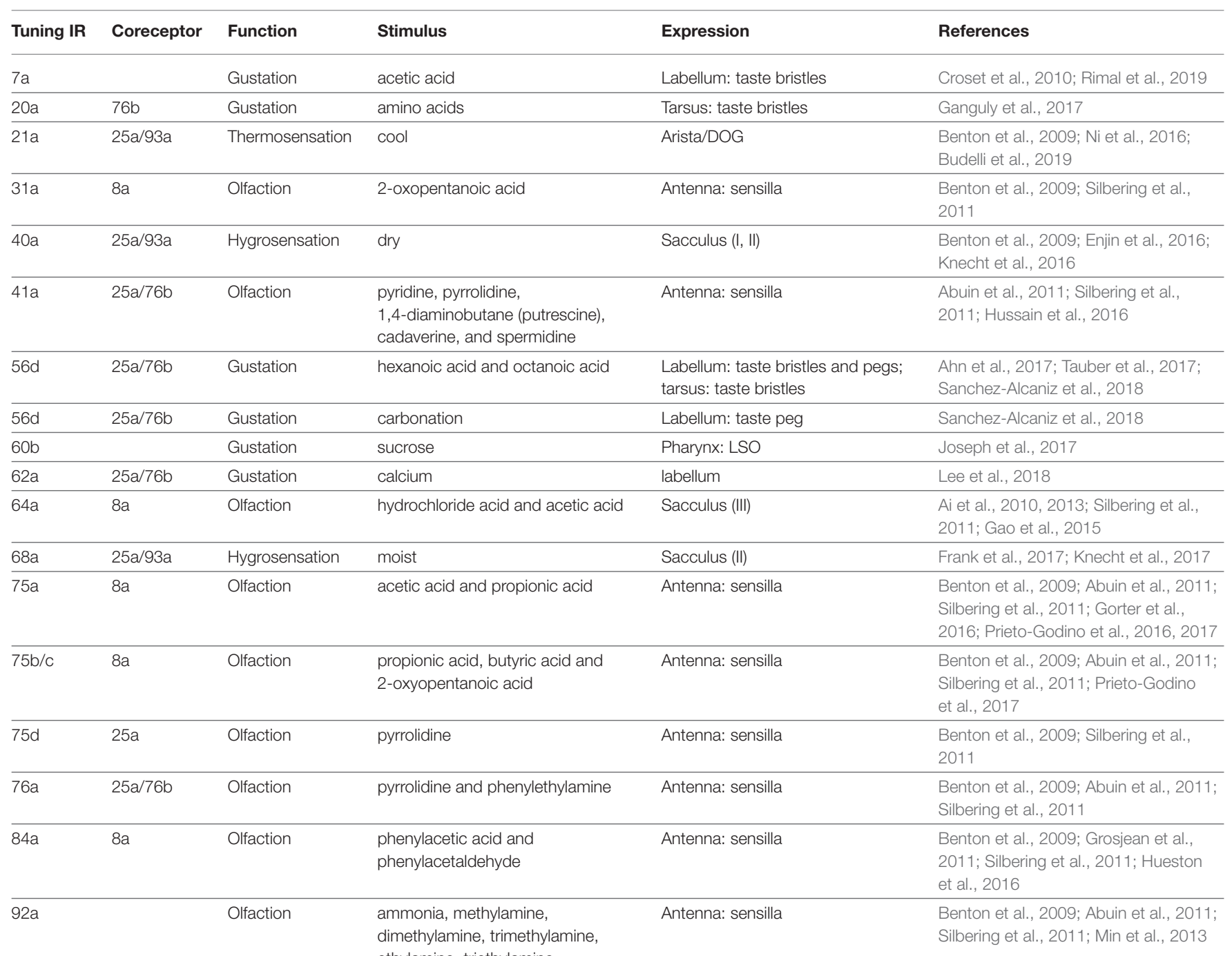




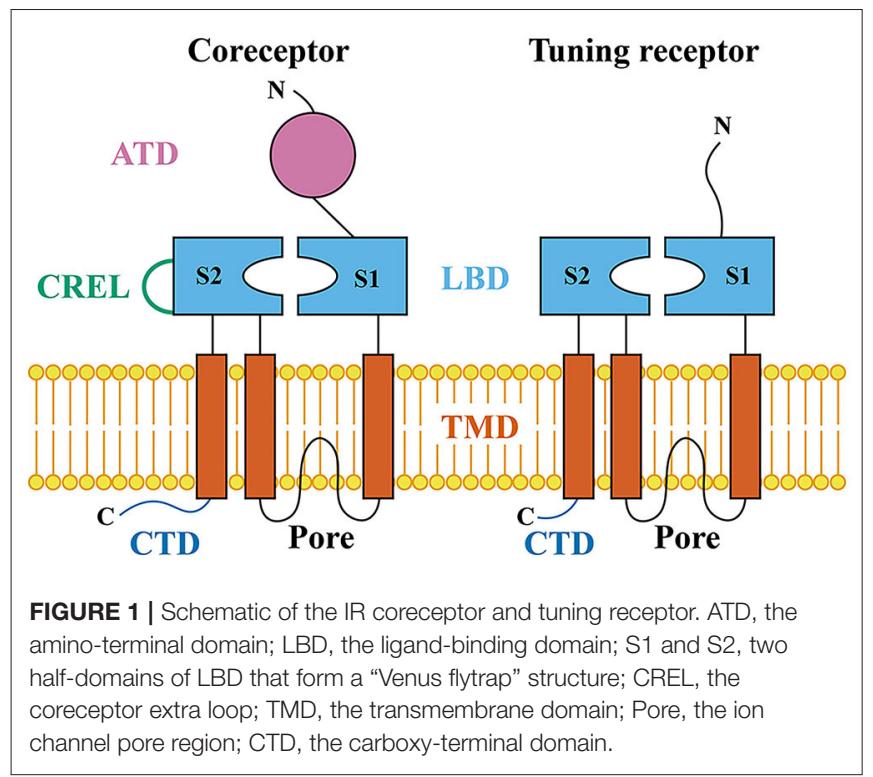

and a re-entrant pore loop (Kuner et al., 2003). S2 is followed by a third transmembrane segment (TM3) and a cytosolic carboxyterminal domain (CTD). The IR coreceptors, IR8a and IR25a, contain the ATD, LBD, TMD, CTD, and coreceptor extra loop (CREL). Tuning IRs and less broadly expressed coreceptors, IR76b and IR93a, do not contain typical ATD domains and CRELs (Figure 1) (Benton et al., 2009; Croset et al., 2010).

The ATD domain is involved in the assembly of iGluR subunits into heteromeric complexes (Jin et al., 2009). The coreceptors, IR8a and IR25a, contain ATD domains (Figure 1) (Croset et al., 2010). Deletion of the ATD in IR8a abolishes the cilia localization, suggesting a role for this domain in protein folding, IR complex assembly, or cilia targeting (Abuin et al., 2011). Moreover, the direct interactions between two IR8a ATDs have been identified by protein fragment complementation assays (Abuin et al., 2019), suggesting that ATDs in coreceptors are involved in the assembly of heteromeric complexes. Tuning IRs and less broad coreceptor IRs, IR76b and IR93a, do not contain typical ATD domains (Figure 1) (Benton et al., 2009; Croset et al., 2010). Instead, they have a relatively short and highly divergent $\mathrm{N}$-terminal region of about 200 amino acids before the LBD S1 domain (Croset et al., 2010; Abuin et al., 2011). Interestingly, deletion of the short amino acid $\mathrm{N}$-terminal region in IR84a also abolishes the normal cilia targeting, suggesting that this fragment has a similar importance to the ATD domain in coreceptors in protein folding, IR complex assembly, or cilia targeting (Abuin et al., 2011). Moreover, the short amino acid Nterminal regions in two IR84a subunits do not interact directly (Abuin et al., 2019).

Both coreceptors and tuning receptors contain the ligandbinding domain (LBD) and transmembrane domain (TMD) (Figure 1) (Benton et al., 2009). The IR LBDs are highly divergent and, thus, the ligand-binding specificity of most or all IRs is likely to be both distinct from that of iGluRs and varied within the IR family (Benton et al., 2009; Croset et al., 2010; Abuin
BOX 1 | Ester, alcohol, amine, and carboxylic acid.

An alcohol carries at least one $\mathrm{OH}$ group bond to a saturated carbon atom. An amine is a derivative of ammonia $\left(\mathrm{NH}_{3}\right)$, wherein one or more hydrogen atoms have been replaced by a substituent.

A carboxylic acid contains a $\mathrm{C}(=\mathrm{O}) \mathrm{OH}$ group attached to an alkyl group. The amino acid and fatty acids are carboxylic acids.

An ester is a derivative of an acid in which at least one $\mathrm{C}(=\mathrm{O}) \mathrm{OH}$ group is replaced by a $\mathrm{C}(=\mathrm{O}) \mathrm{OR}$ group. $\mathrm{R}$ represents an alkyl group.

A ketone contains a $\mathrm{C}(=\mathrm{O})$ group bonded to two alkyl groups.

et al., 2011). For example, all iGluRs have an arginine (R) residue in $\mathrm{S} 1$ that binds the glutamate $\alpha$-carboxyl group $(\mathrm{C}(=\mathrm{O}) \mathrm{OH})$; only 19 IRs retain this residue. Moreover, a threonine (T) residue in the first half of S2 exists in all AMPA and kainate receptors and functions to contact the glutamate $\gamma$-carboxyl group. Only 9 IRs retain this residue. In the second half of S2, all iGluRs have an aspartate (D) or glutamate (E) that interacts with the glutamate $\alpha$-amino group $\left(\mathrm{NH}_{2}\right)$. Only 10 IRs retain this conserved residue. Importantly, only three IRs (IR8a, IR75a, and IR75c) retain all three conserved residues ( $R, T$, and $\mathrm{D} / \mathrm{E})$. These conserved residues, at least in IR8a, are unlikely to recognize glutamate by the mutational analysis of IR8a (Mayer, 2006; Benton et al., 2009; Abuin et al., 2011).

The LBD domains are important for receptor targeting and chemical recognition. Mutation of the conserved $\mathrm{R}$ and $\mathrm{D}$ in IR8a reduces or abolishes the efficiency of targeting the cilia, suggesting a role for the IR8a LBD in receptor localization (Abuin et al., 2011). IR84a retains the $\mathrm{R}$ residue in the $\mathrm{S} 1$ domain. A point mutation of this residue does not affect the receptor targeting to cilia, but eliminates the ligand-evoked responses, indicating that the IR84a LBD is required for chemical recognition (Abuin et al., 2011). Interestingly, this $\mathrm{R}$ residue is conserved in acidsensing IRs (IR31a, IR64a, IR75a, IR75b, IR75c, and IR84a) but divergent in amine-sensing IRs (IR41a, IR76a, and IR92a) (Box 1; Table 1), suggesting that this $\mathrm{R}$ residue might play a conserved role between iGluRs and acid-sensing IRs in conjugating the carboxyl group $(\mathrm{C}(=\mathrm{O}) \mathrm{OH})$ of their ligands (Benton et al., 2009; Silbering et al., 2011). Studies on IR75a in different Drosophila species provide additional evidence about the function of the LBD domain in chemical recognition. Mutations of three amino acids (T289, Q536, and F538) in the IR75a LBD domain changes the responses to acetic acid, butyric acid, and 2-oxopentanoic acid (Box 2), suggesting one or more of these residues act as chemical-specificity determinants (Prieto-Godino et al., 2016).

The coreceptor extra loop (CREL) is highly conserved in IR8a and IR25a across the insect and Protostomia, respectively. This loop is absent in tuning IRs or iGluRs (Figure 1). CRELs locate near the beginning of the S2 domain and possess several conserved characteristics, including the presence of predicted short alpha-helical and beta-sheet regions and a single consensus N-glycosylation target motif (NXS/T) (Abuin et al., 2019). Deletion of CREL or mutation of the N-glycosylation site in 


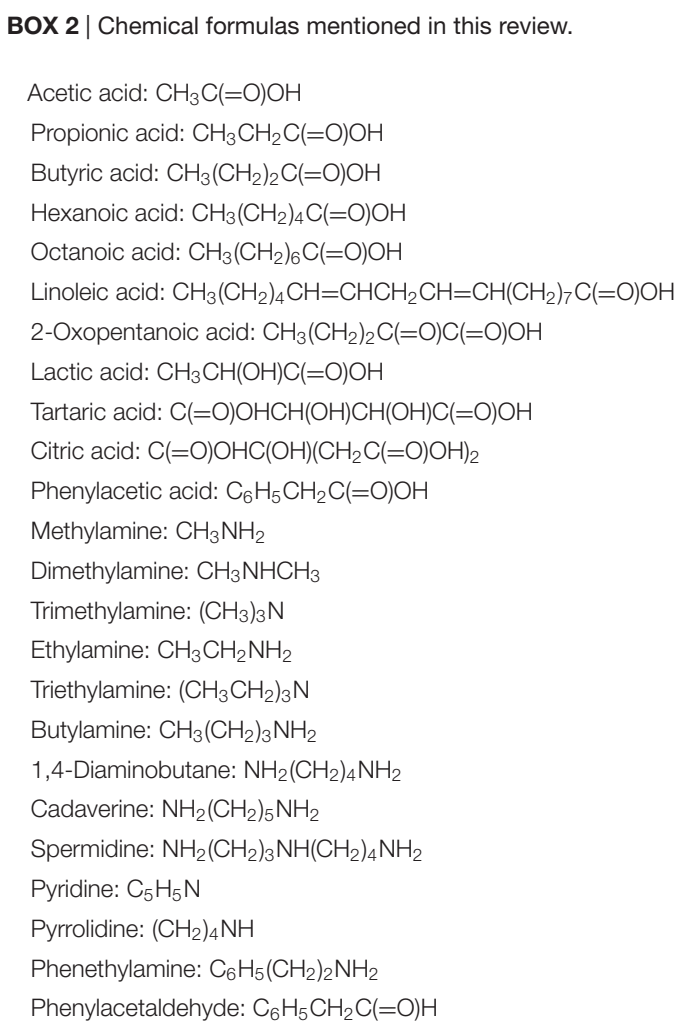

CREL affects the cilia trafficking of the IR complex, suggesting a role of CREL in receptor targeting (Abuin et al., 2019).

The ion channel pore is the most conserved region between IRs and iGluRs (Benton et al., 2009; Croset et al., 2010). To confirm that IRs act as ion channels, multiple point mutations in the putative ion channel pore have been generated. In the pore region of GluA2, glutamine is required to control calcium permeability (Hume et al., 1991). In the same position, IR84a retains glutamine $(\mathrm{Q} 401)$. The IR84a mutant receptors that have a point mutation of this glutamine do not affect the conductance of monovalent cations but lack calcium permeability (Abuin et al., 2011; Ng et al., 2019). Moreover, IR8a contains a proline in the pore region. A point mutation of this proline decreases the ligand-evoked currents, affects the conductance of monovalent cations, and abolishes the calcium-dependent conductance (Abuin et al., 2011). These data together support the notion that IRs function as ion channels and suggest that the pore regions of both coreceptors and tuning receptors are required to mediate the ionic pathway.

TM3 is also involved in controlling the ion conductance of iGluRs (Sobolevsky et al., 2009). In the absence of ligands, iGluRs are closed. A spontaneous A288T mutation in TM3 disrupts the closed conformation, resulting in a constitutive sodium conductance (Zuo et al., 1997). When expressing in HEK293T cells, IR76b behaves as a sodium leaky channel and is in a constitutively open state. This constitutive current can

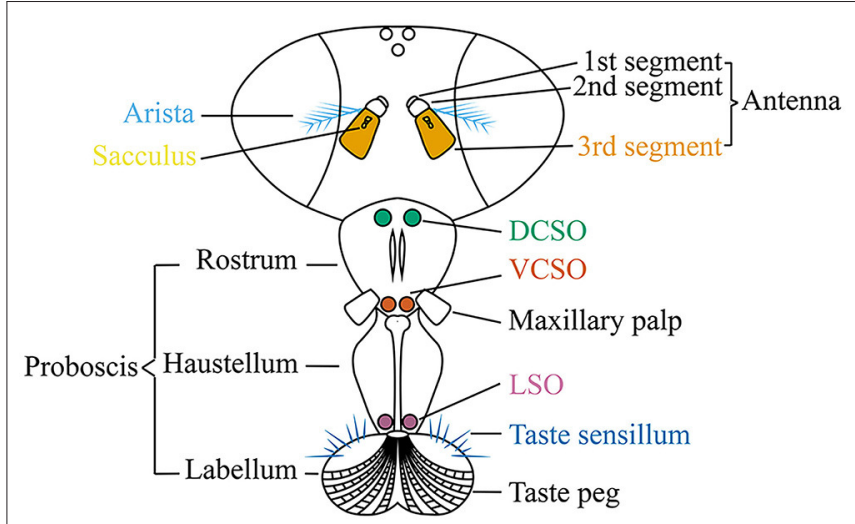

FIGURE 2 | The adult fly head to show the olfactory organs (the third antennal segment and maxillary palp), gustatory organs [the labellar taste sensillum, labellar taste peg, labial sense organ (LSO), ventral cibarial sense organ (VCSO), and dorsal cibarial sense organ (DCSO)], thermosensory organ (the arista), and hygrosensory organ (the sacculus).

be attenuated by a $\mathrm{T}$ to $\mathrm{A}$ point mutation in the corresponding position of IR76b (Zhang et al., 2013). This point mutation also blocks the in vivo low salt responses (Zhang et al., 2013).

While deletion of the CTD (Figure 1) of IR84a does not affect localization or function, deletion of the CTD of IR8a strongly reduces cilia-targeting efficiency (Abuin et al., 2011), suggesting a role of the coreceptor CTD domains in receptor localization.

Interestingly, when IR8a is the coreceptor in an IR complex, the complex often contains a single tuning receptor and, thus, two IR8a subunits and a couple of the same tuning receptors are in the complex (Abuin et al., 2011, 2019). However, IR25a often complexes with IR76b to mediate chemical sensation and IR93a to mediate temperature and humidity sensation (Table 1). Studies about the structure of IR complexes that include IR25a, IR76b, and IR93a have not been reported.

\section{CHEMOSENSATION}

Animals mainly depend on their smell and taste to assess the quality and nutritional value of food. While olfaction plays a key role for insects to identify food, gustation is crucial for the decision of whether to feed. Moreover, both olfaction and gustation are important for courtship behavior (Squire et al., 2003; Montell, 2009; Carey and Carlson, 2011).

\section{Olfaction}

Olfactory sensory organs in adult flies are housed in the third antennal segment and maxillary palp (Figure 2). Each olfactory sensory neuron (OSN) is located in a sensillum and expresses a specific type or very small combination of receptors. All OSNs that express the same receptor converge their axons to a single pair of glomeruli in the antennal lobe (Vosshall and Stocker, 2007). Upon additional local processing at the level of the antennal lobe, the odor information is sent via projection neurons to two main higher brain centers, the mushroom body and the lateral horn (Masse et al., 2009). 
The third antennal segment bears three classes of olfactory sensilla: basiconic, trichoid, and coeloconic sensilla. Each sensillum contains the dendrites of up to three OSNs with different odorant response profiles. Three classes of olfactory molecular receptors have been identified: the odorant receptors (ORs), ionotropic receptors (IRs), and a few gustatory receptors (GRs) (Vosshall et al., 2000; Jones et al., 2007; Benton et al., 2009). OSNs innervating basiconic and trichoid sensilla generally express ORs and respond to many esters, ketones, and alcohols; while, except for OR35a-expressing neurons, OSNs housed in coeloconic sensilla express IRs and respond to many amines and carboxylic acids (Box 1) (de Bruyne et al., 2001; Hallem et al., 2004; Yao et al., 2005; van der Goes van Naters and Carlson, 2007; Vosshall and Stocker, 2007; Benton et al., 2009; Silbering et al., 2011). Coeloconic sensilla can be classified into four clusters (ac1, ac2, ac3, and ac4) by their expression of stereotyped combinations of IR genes (Benton et al., 2009). IRs are also expressed in the sacculus, a three-chambered pit under the antennal surface, and in the arista, a feather-like structure that extended from the third segment of the antenna (Foelix et al., 1989; Shanbhag et al., 1995) (Figure 2). Although OR- and IR-expressing OSNs project to complementary sets of glomeruli in the antennal lobe, the axon terminals of the projection neurons postsynaptic to IR or OR OSNs are interdigitated within the mushroom body and the lateral horn (Silbering et al., 2011).

Three IR coreceptors, IR8a, IR25a, and IR76b, function in olfactory systems. IR8a is broadly expressed in the main portion of the third antennal segment and the third chamber of the sacculus (Supplementary Table 1) (Benton et al., 2009; Abuin et al., 2011; Ai et al., 2013; Menuz et al., 2014). IR8a is not detected elsewhere in the adult head, body, appendages, or larval stages (Benton et al., 2009; Sanchez-Alcaniz et al., 2018). IR8a is a coreceptor, generally forming heterotetrameric complexes with tuning IR receptors to detect carboxylic acids (Box 1) (Silbering et al., 2011). IR8a in mosquitoes is also necessary for sensing acidic volatiles, such as lactic acid, and human odor (Box 2) (Raji et al., 2019).

IR25a is the IR gene that is most similar to the ancestral IR and evolved from a bilaterian non-NMDA receptor gene (Croset et al., 2010). It is another coreceptor that is broadly expressed in the main portion of the third antennal segment (Benton et al., 2009; Abuin et al., 2011; Chen et al., 2015). Unlike IR8a, IR25a is expressed in the first and second saccular chambers and arista (Benton et al., 2009; Abuin et al., 2011; Chen et al., 2015; Steck et al., 2018; Budelli et al., 2019). IR25a is also found in the second antennal segment (Chen et al., 2015), proboscis (Chen and Amrein, 2017; Chen and Dahanukar, 2017; Lee et al., 2018; Sanchez-Alcaniz et al., 2018; Steck et al., 2018), legs (Chen et al., 2015; Chen and Amrein, 2017; Lee et al., 2018; Sanchez-Alcaniz et al., 2018; Steck et al., 2018), wings (Sanchez-Alcaniz et al., 2018), and abdomen (Sanchez-Alcaniz et al., 2018) in adults (Supplementary Table 1). In larvae, IR25a is broadly expressed (Supplementary Table 2) (Stewart et al., 2015; Croset et al., 2016; van Giesen et al., 2016; Rist and Thum, 2017; Sanchez-Alcaniz et al., 2018). As an olfactory receptor, IR25a detects amines when forming heterotetrameric complexes with tuning IR receptors
(Box 1) (Silbering et al., 2011). Generally, IR25a and IR8a have selective functional properties and cannot substitute each other (Abuin et al., 2011).

IR76b is the third identified coreceptor in the olfactory system that is expressed in the main portion of the third antennal segment, but not the sacculus or arista (Benton et al., 2009; Menuz et al., 2014; Hussain et al., 2016; Steck et al., 2018). IR76b is also broadly expressed outside of the olfactory system, including the proboscis (Zhang et al., 2013; Hussain et al., 2016; Chen and Amrein, 2017; Chen and Dahanukar, 2017; Ganguly et al., 2017; Jaeger et al., 2018; Lee et al., 2018; Sanchez-Alcaniz et al., 2018; Steck et al., 2018), legs (Zhang et al., 2013; Hussain et al., 2016; Chen and Amrein, 2017; Ganguly et al., 2017; Lee et al., 2018; Sanchez-Alcaniz et al., 2018; Steck et al., 2018), wings (Zhang et al., 2013; Hussain et al., 2016; Sanchez-Alcaniz et al., 2018; Yanagawa et al., 2019), and abdomen (Supplementary Table 1) (SanchezAlcaniz et al., 2018). Moreover, IR76b is detected in larvae (Supplementary Table 2) (Stewart et al., 2015; Croset et al., 2016; Rist and Thum, 2017; Sanchez-Alcaniz et al., 2018). In olfactory systems, IR76b often complexes with IR25a to detect variant odorants. Unlike IR8a or IR25a, IR76b does not contain the ATD or CREL domain.

IR31a is expressed in the OSNs that project to antennal ac1 coeloconic sensilla (Benton et al., 2009). Their axons terminate at VL2p glomeruli in the antennal lobe (Silbering et al., 2011). The coreceptor, IR8a, is necessary for the function of IR31a neurons to respond to 2-oxopentanoic acid (Box 2; Table 1) (Silbering et al., 2011).

IR41a is expressed in the OSNs whose dendrites are housed in antennal ac2 coeloconic sensilla (Silbering et al., 2011). Their axons are projected to VC5 glomeruli (Silbering et al., 2011; Hussain et al., 2016). The IR4la positive OSNs respond to amine ligands, including pyridine, pyrrolidine, 1,4-diaminobutane (putrescine), cadaverine, and spermidine (Box 2) (Silbering et al., 2011; Hussain et al., 2016). IR25a and IR76b are coexpressed with IR41a and necessary for the amine responses (Table 1) (Abuin et al., 2011; Silbering et al., 2011; Hussain et al., 2016).

IR64a is expressed in the OSNs surrounding the third saccular chamber (Benton et al., 2009; Ai et al., 2010). These OSNs send their axons to two pairs of glomeruli, DC4 and DP1m (Ai et al., 2010, 2013; Silbering et al., 2011; Gao et al., 2015). The dendrites of DC4- and DP1m-targeting OSNs innervate ventral and dorsal compartments of the third saccular chamber, respectively (Ai et al., 2013). IR8a is expressed in both DC4- and DP1m-targeting IR64a positive neurons (Ai et al., 2013). DC4-targeting OSNs detect hydrochloric acid $(\mathrm{HCl})$ and acetic acid (Box 2). IR8a and IR64a form an acid receptor that is necessary and sufficient for acid responses (Table 1) (Ai et al., 2010, 2013; Silbering et al., 2011; Gao et al., 2015).

IR75a is expressed in the ac2/3 coeloconic OSNs. The axons of ac2 OSNs target DP11 glomeruli, while ac3 axons target DL2 (Benton et al., 2009; Silbering et al., 2011; Prieto-Godino et al., 2016, 2017). IR75a forms an acidic receptor with IR8a and is necessary and sufficient to detect acetic acid and propionic acid (Box 2) (Abuin et al., 2011; Silbering et al., 2011; Gorter et al., 2016; Prieto-Godino et al., 2016). IR75b and IR75c are expressed 
in the ac3I and ac3II coeloconic OSNs, respectively (PrietoGodino et al., 2017). Both IR75b and IR75c are sufficient to respond to propionic acid, butyric acid, and 2-oxyopentanoic acid (Box 2) with different sensitivities. These responses also depend on IR8a (Table 1) (Benton et al., 2009; Abuin et al., 2011; Silbering et al., 2011; Prieto-Godino et al., 2017).

IR75d is expressed in the ac1/2/4 coeloconic OSNs that send their axons to VL1 glomeruli (Benton et al., 2009; Silbering et al., 2011). IR75d-expressing OSNs respond to pyrrolidine and these responses are IR25a dependent (Box 2; Table 1) (Silbering et al., 2011).

IR76a is expressed in the ac4 coeloconic OSNs that project to VM4 (Benton et al., 2009; Silbering et al., 2011). IR76a and two coreceptors, IR25a and IR76b, are sufficient to detect pyrrolidine and phenylethylamine (Box 2; Table 1) (Abuin et al., 2011; Silbering et al., 2011).

IR84a is expressed in the ac4 coeloconic OSNs that send their axons to VL2a (Benton et al., 2009; Grosjean et al., 2011; Silbering et al., 2011; Hueston et al., 2016). Two IR8a subunits and two IR84a subunits form a heterotetramer that is necessary and sufficient to detect phenylacetic acid and phenylacetaldehyde (Box 2; Table 1) (Benton et al., 2009; Abuin et al., 2011; Grosjean et al., 2011; Silbering et al., 2011). VL2a is one of the only three glomeruli that are larger in males and whose inputs and outputs express male-specific isoforms of the behavioral sex determination gene fruitless (Manoli et al., 2005; Stockinger et al., 2005). Although IR84a does not show sexually dimorphic expression and is not tuned to fly-derived pheromones, it increases male courtship in the presence of IR84a ligands, such as phenylacetic acid (Grosjean et al., 2011).

IR92a is expressed in the ac1 coeloconic OSNs that projects to VM1 (Benton et al., 2009; Silbering et al., 2011). IR92a is necessary to detect ammonia, methylamine, dimethylamine, trimethylamine, ethylamine, triethylamine, butylamine, and pyrrolidine (Box 2; Table 1) (Silbering et al., 2011; Min et al., 2013). Although IR92a is found to be coexpressed with IR8a, IR25a, and IR76b, none of these coreceptors are necessary for the responses of ammonia or other amines (Abuin et al., 2011; Min et al., 2013).

IR25a and IR76b have been identified in the larval olfactory system, the dorsal organ ganglion (DOG) (Supplementary Table 2) (Stewart et al., 2015; van Giesen et al., 2016; Sanchez-Alcaniz et al., 2018). However, their functions in larval olfaction have not been demonstrated.

\section{Gustation}

Gustatory receptor neurons (GRNs) in adult flies are found in many peripheral organs, including the labellum, pharynx, legs, wing margins, and abdomen (Figure 2). The labellum contains taste sensilla and taste pegs. The pharynx contains three organs: the labial sense organ (LSO), ventral cibarial sense organ (VCSO), and dorsal cibarial sense organ (DCSO). These pharyngeal taste organs are critical to monitor food quality. The head of the Drosophila larva contains three external chemosensory organs: the dorsal organ ganglion (DOG), terminal organ ganglion (TOG), and ventral organ ganglion (VOG) (Figure 3). In the

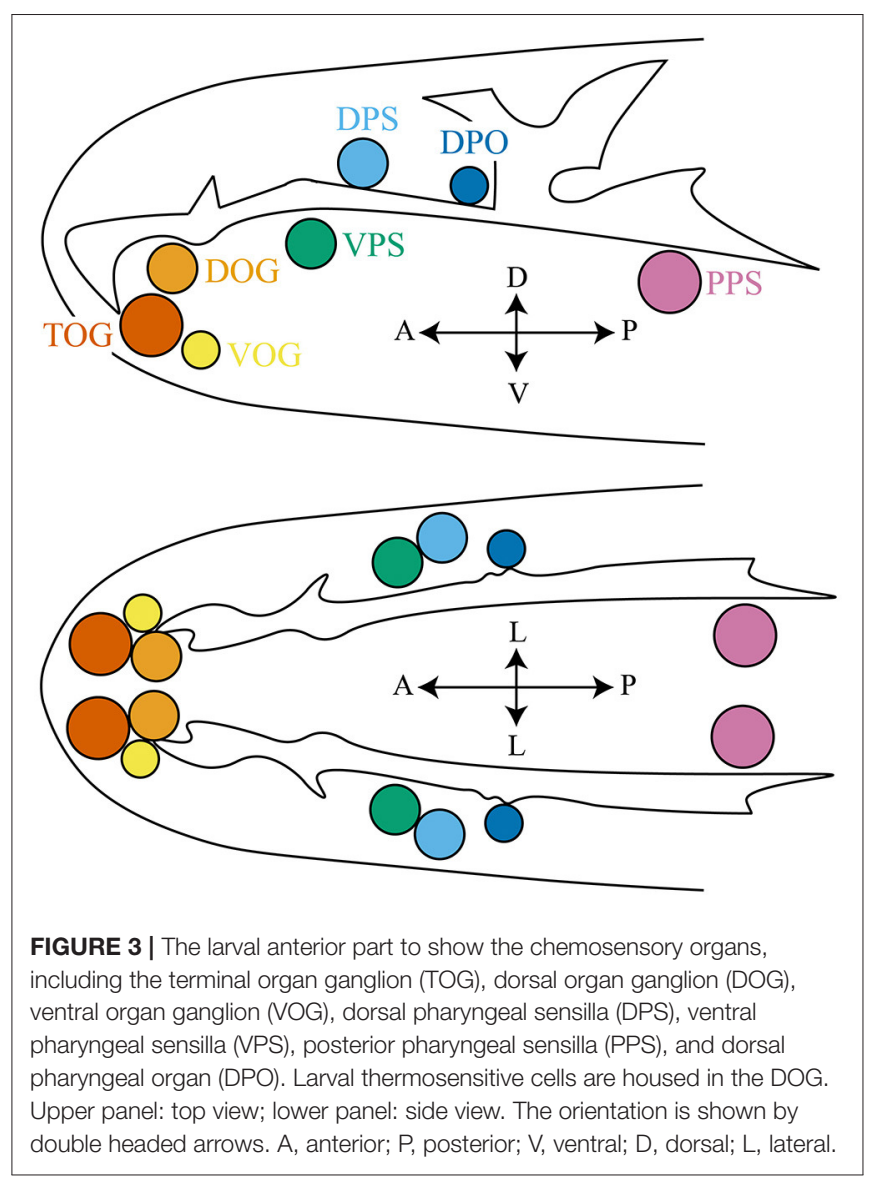

pharynx, there are four internal chemosensory organs: the dorsal, ventral, and posterior pharyngeal sensilla (DPS, VPS, and PPS), and dorsal pharyngeal organ (DPO). Moreover, the posterior tip of the larva also contains chemosensory neurons. GRNs are housed in sensilla that have a single pore for tastants to enter (Montell, 2009). IRs have been found in all identified taste organs (Supplementary Tables 1, 2). Unlike IRs and ORs which are expressed in complementary OSNs, IRs and GRs are coexpressed in GRNs (Koh et al., 2014).

IR25a and IR76b, not IR8a, function as gustatory coreceptors to detect different tastants. They are broadly expressed in all taste organs in both larval and adult flies, with the exception that IR76b is not detected in the posterior tip of the larva (Supplementary Tables 1, 2) (Zhang et al., 2013; Chen et al., 2015; Stewart et al., 2015; Croset et al., 2016; Hussain et al., 2016; van Giesen et al., 2016; Chen and Amrein, 2017; Chen and Dahanukar, 2017; Ganguly et al., 2017; Rist and Thum, 2017; Jaeger et al., 2018; Lee et al., 2018; Sanchez-Alcaniz et al., 2018; Steck et al., 2018; Yanagawa et al., 2019). As in olfactory systems, IR25a and IR76b often collaboratively mediate responses to tastants. For example, both IR25a and IR76b are necessary to detect acids in tarsal GRNs, including acetic acid, citric acid, tartaric acid, hexanoic acid, octanoic acid, linoleic acid, and hydrochloric acid (Box 2) (Ahn et al., 2017; Chen and Amrein, 
2017). In labellar taste bristles, IR25a and IR76b are necessary for responses to salt (high and low), potassium chloride, and calcium (Jaeger et al., 2018; Lee et al., 2018). They can also function independently in mediating responses to other tastants. For example, IR25a, not IR76b, is necessary to detect denatonium, a bitter tastant, in the C7 GRN in the TO (van Giesen et al., 2016). On the other hand, IR76b but not IR25a is necessary to detect the yeast/amino acid mixture in the tarsal bristles and larval TO (Croset et al., 2016; Ganguly et al., 2017). Labellar IR76b is necessary to detect polyamines, including 1,4-diaminobutane and cadaverine (Box 2), and to mediate avoidance of polyamines (Hussain et al., 2016). Moreover, IR76b alone is sufficient to form the low salt receptor that does not require additional IRs (Zhang et al., 2013). IR76b in the labellum has also been proposed as a sensitivity modulator to regulate the preference for acetic acid, citric acid, and sucrose (Box 2) (Chen H. L. et al., 2019).

IR7a is expressed in the bitter GRNs in the labellum and is required for the avoidance of acetic acid (Box 2) (Rimal et al., 2019). Although IR25a is coexpressed with IR7a, it is not necessary for the function of IR7a in detecting acetic acid (Table 1) (Croset et al., 2010; Rimal et al., 2019). IR7a and IR7f are expressed in the blood-sensitive GRNs on the stylet tip of female mosquitoes (Jové et al., 2020).

IR20a in tarsal GRNs is coexpressed with IR76b and, at least partially, required for detecting amino acid mixture (Table 1) (Ganguly et al., 2017). Since the loss of IR20a results in weaker defects in amino acid detection, IR20a may be functionally redundant with other IRs (Ganguly et al., 2017). Moreover, expression of IR20a is sufficient to confer sensitivities of amino acid mixture to labellar sensilla and to block the function of IR76b in detecting low salt (Zhang et al., 2013; Ganguly et al., 2017).

IR52a is expressed in the adult legs and wings (Koh et al., 2014; He et al., 2019). It shows sexual dimorphic expression and is necessary for sexual behavior in both male and female flies (Koh et al., 2014; He et al., 2019). IR52c and IR52d are expressed in sexually dimorphic taste neurons in forelegs. These neurons are activated by contacting females during courtship (Koh et al., 2014). The function of IR52a, IR52c, and IR52d may depend on IR25a and IR76b, since IR25a and IR76b are coexpressed with IR52a, IR52c, and IR52d in forelegs (Koh et al., 2014). However, the chemical cues that activate IR52a, IR52c, and IR52d have not been found.

IR56d is found in labellar taste sensilla and pegs, as well as tarsal bristles (Ahn et al., 2017; Tauber et al., 2017; SanchezAlcaniz et al., 2018). Together with IR25a and IR76b, IR56d is necessary for the responses to multiple fatty acids, including hexanoic acid, octanoic acid, and linoleic acid (Box 2; Table 1) (Ahn et al., 2017; Tauber et al., 2017; Sanchez-Alcaniz et al., 2018). Interestingly, the norpA gene, which encodes a phospholipase $\mathrm{C}$ (PLC) that acts as a downstream effector of G-protein coupled receptors in the fly's visual system, is necessary for these responses (Ahn et al., 2017; Tauber et al., 2017). These three IRs are also necessary to detect carbonations in taste pegs (Table 1) (SanchezAlcaniz et al., 2018).

IR60b mediates sucrose responses in LSO in the adult pharynx and acts in limiting sucrose consumption (Table 1) (Koh et al., 2014; Joseph et al., 2017; Chen Y. D. et al., 2019).
IR62a forms a calcium-responsive receptor with IR25a and IR76b in the labellum and is necessary to mediate calcium avoidance (Table 1) (Lee et al., 2018).

\section{THERMOSENSATION AND HYGROSENSATION}

Temperature and humidity influence the fitness and geographic distribution of all animals and are crucial for insects. Insects rely on the ambient temperatures to set their body temperatures and must avoid dehydration, a constant threat for insects due to their small bodies and large ratios of surface area to volume (Garrity et al., 2010; Chown et al., 2011).

IRs function in thermosensation and hygrosensation. In adult flies, IR-formed thermoreceptors have been identified in the arista and sacculus (Figure 2), although the molecular basis of the saccular thermoreceptors has not been resolved (Enjin et al., 2016; Frank et al., 2017; Budelli et al., 2019). The sacculus also houses hypersensitive neurons that depend on IRs to detect the humidity change (Enjin et al., 2016; Knecht et al., 2016, 2017; Frank et al., 2017). While the IR-formed humidity receptors have not been found in larvae, the IR-formed thermoreceptors have been identified in the larval dorsal organ ganglion (DOG) (Figure 3) (Knecht et al., 2016; Ni et al., 2016).

As discussed, IR25a is broadly expressed and functions as a coreceptor for olfaction and gustation to detect variant chemicals. In addition, IR25a is also expressed in thermoresponsive neurons in the larval DOG and adult arista to support thermosensation, as well as humidity sensitive neurons in the sacculus to support hygrosensation (Figures 2, 3; Supplementary Tables 1, 2) (Benton et al., 2009; Enjin et al., 2016; Knecht et al., 2016, 2017; Ni et al., 2016; Frank et al., 2017; Budelli et al., 2019). Moreover, IR25a in the femur chordotonal organ neurons is proposed to be a warm receptor to mediate circadian clock resetting by temperature, although the tuning receptors, if any, for this process are still unknown (Chen et al., 2015). In the brain, IR25adependent sensory neurons with different sensory modalities send their projections to different regions where the specific sensory information is processed. For example, the antennal IR25a-positive sensory neurons project to distinct glomeruli based on the specific tuning receptors. The IR41a- and IR76adependent OSNs send their axons to two different glomeruli, VC5 and VM4 (Benton et al., 2009; Silbering et al., 2011; Hussain et al., 2016). The aristal IR21a cool cells project to the VP3 glomerulus (Silbering et al., 2011; Marin et al., 2020). The VP4 and VP5 glomeruli receive information from IR40a dry cells and IR68a moist cells, respectively (Enjin et al., 2016; Knecht et al., 2016, 2017; Frank et al., 2017; Marin et al., 2020). In contrast, the IR25a-positive GRNs project to the sub-esophagael zone (SEZ), the primary taste center of the Drosophila central nervous system (Tauber et al., 2017; Sanchez-Alcaniz et al., 2018). Besides IR25a, IR93a also functions as a coreceptor specific for thermosensation and hygrosensation (Enjin et al., 2016; Knecht et al., 2016, 2017; Frank et al., 2017; Budelli et al., 2019).

The tuning receptor for cool detection is IR21a (Ni et al., 2016; Budelli et al., 2019). IR21a is expressed in the larval DOG 
and adult arista (Figures 2, 3). It forms a cool-active receptor with IR25a and IR93a and is necessary and sufficient to mediate the cool responsiveness (Table 1) (Ni et al., 2016; Budelli et al., 2019). These three IRs are also critical for the morphogenesis of the membrane-riched dendritic structure in cooling cells (Budelli et al., 2019). Although IR21a is expressed in the sacculus (Benton et al., 2009), the coreceptors and functional importance have not been identified. Moreover, IR21a in mosquitoes is necessary for host-seeking behavior (Greppi et al., 2020).

IR40a and IR68a are the tuning receptors for dry and moist detection, respectively (Enjin et al., 2016; Knecht et al., 2016, 2017; Frank et al., 2017). IR40a is expressed in the first and second saccular chambers and necessary for the dry activation of dry neurons (Figure 2; Table 1) (Benton et al., 2009; Enjin et al., 2016; Knecht et al., 2016). IR68a is mainly expressed in the second chamber of the sacculus and necessary for detecting moist air (Figure 2; Table 1) (Frank et al., 2017; Knecht et al., 2017). The function of IR40a and IR68a also depends on IR25a and IR93a (Table 1) (Enjin et al., 2016; Knecht et al., 2016, 2017; Frank et al., 2017).

\section{DISCUSSION}

In Drosophila melanogaster, 63 IRs have been identified and function in olfaction, gustation, thermosensation, and hygrosensation (Benton et al., 2009; Koh et al., 2014; van Giesen and Garrity, 2017). The expression of most IRs-except for IR48a, IR51a, IR54a, IR60a, and IR87a-has been shown by in situ hybridization, antibody staining, and/or driver lines (Supplementary Tables 1, 2). Besides four coreceptors (IR8a, IR25a, IR76b, and IR93a), the functions of only 19 tuning receptors have been identified (Table 1). IR52a, IR52c, and IR52d are expressed in taste neurons in wing margins and legs. They are required for courtship behavior (Koh et al., 2014; He et al., 2019). However, the ligands of these IRs are unknown and, thus, are not listed in Table 1. To comprehensively understand the IR family and peripheral sensory systems in Drosophila melanogaster, it is important to identify the function of each IR. Moreover, the IR family is a highly divergent subfamily of iGluRs (Benton et al., 2009). Although iGluRs have been well-studied, it is not fully understood how IR-formed receptors are gated by their chemical ligands. Most importantly, how do IR-formed receptors sense temperature and humidity change?

In addition, a tuning receptor usually binds one or two coreceptors to detect a specific environmental stimulus. However, although none of IR8a, IR25a, and IR76b is necessary, IR92amediated responses of ammonia can only be detected when IR92a is expressed in IR OSNs, but not OR OSNs, in the antenna (Abuin et al., 2011; Min et al., 2013), suggesting these three coreceptors might have redundant functions in supporting the IR92a-dependent responses of ammonia. Thus, it is worth testing whether a tuning IR can pair with different coreceptors to form a functional receptor and, if so, whether these receptors have the same function.

IRs are conserved across protostome species and bioinformatic analyses of $I R$ genes have been performed in many species (Benton et al., 2009; Croset et al., 2010; Bengtsson et al., 2012; Glaser et al., 2013; Poivet et al., 2013; Rytz et al., 2013; Cao et al., 2014; Groh-Lunow et al., 2014; Liu et al., 2014, 2017, 2018; Missbach et al., 2014; Ahmed et al., 2016; Dippel et al., 2016; Macharia et al., 2016; van Schooten et al., 2016; Cicconardi et al., 2017; Latorre-Estivalis et al., 2017; Yang et al., 2017, 2020; Zbinden et al., 2017; Harrison et al., 2018; Kozma et al., 2018; Matthews et al., 2018; Robertson et al., 2018, 2019; Rojas et al., 2018; Andersson et al., 2019; Lei et al., 2019; Li et al., 2019; Ma et al., 2019; Xu et al., 2019; Balart-García et al., 2020; Sun et al., 2020; Wu et al., 2020; Yin et al., 2020). However, the functional studies of IRs in non-model organisms are largely lacking. This is mainly due to the limitation of genetic approaches in these organisms. To overcome this problem, two genetic approaches have been applied. First, $I R$ genes from non-model organisms are cloned and expressed in Drosophila melanogaster to perform functional studies. For example, the functional specificities of Drosophila sechellia IR75a, IR75b, and IR75c are firstly obtained by expressing these genes in Drosophila melanogaster (PrietoGodino et al., 2016, 2017). Moreover, expression of the mosquito IR76b is sufficient to rescue the defects in detecting amino acid mixture in Ir76b mutant flies (Ganguly et al., 2017). The IR25a and IR93a orthologues in honey bee parasitic mites, Tropilaelaps mecedease, are sufficient to rescue the temperature and humidity preference defects when expressing in IR25a and IR93a mutant flies, respectively (Lei et al., 2019). These data suggest conserved roles of IR76b, IR25a, and IR93a in sensory function, although the endogenous function is not evaluated. Second, with the advance of CRISPR techniques, genetic manipulation is achieved in many non-model organisms. For instance, the functional necessity of IR8a, IR75b, and IR64a in noni attraction is studied in Drosophila sechellia by generating the corresponding mutants using CRISPR techniques (Auer et al., 2020). In mosquitoes, the IR21a mutants have been generated by CRISPR techniques and its necessity for cool responses in thermosensory neurons in the antennal tip and heat-seeking behavior has been identified (Greppi et al., 2020). The IR8a mutant mosquitoes are also generated using CRISPR techniques. These mutant mosquitoes are fundamental to identify the necessity of IR8a in sensing human odor during blood feeding (Raji et al., 2019). The driver lines of $I R 7 a$ and IR7f are expressed in the blood-sensitive GRNs on the stylet tip on female mosquitoes, although their contribution to blood ligand detection is unknown (Jové et al., 2020). Functional studies of IRs in different animals will play a key role in understanding molecular mechanisms of multiple sensory modalities in various disease vectors and pests and, thus, help to develop tools to control them.

\section{AUTHOR CONTRIBUTIONS}

LN drafted the initial manuscript and approved the final version of the manuscript.

\section{FUNDING}

This work was funded by NIH R01GM140130 and NIH R21MH122987 to LN. 


\section{ACKNOWLEDGMENTS}

I acknowledge Alisa A. Omelchenko and Ainul Huda for comments on the manuscript.

\section{REFERENCES}

Abuin, L., Bargeton, B., Ulbrich, M. H., Isacoff, E. Y., Kellenberger, S., and Benton, R. (2011). Functional architecture of olfactory ionotropic glutamate receptors. Neuron 69, 44-60. doi: 10.1016/j.neuron.2010. 11.042

Abuin, L., Prieto-Godino, L. L., Pan, H., Gutierrez, C., Huang, L., Jin, R., et al. (2019). In vivo assembly and trafficking of olfactory Ionotropic Receptors. BMC Biol. 17:34. doi: 10.1186/s12915-019-0651-7

Ahmed, T., Zhang, T., Wang, Z., He, K., and Bai, S. (2016). Gene set of chemosensory receptors in the polyembryonic endoparasitoid Macrocentrus cingulum. Sci. Rep. 6:24078. doi: 10.1038/srep24078

Ahn, J. E., Chen, Y., and Amrein, H. (2017). Molecular basis of fatty acid taste in Drosophila. Elife 6:e30115. doi: 10.7554/eLife.30115.034

Ai, M., Blais, S., Park, J. Y., Min, S., Neubert, T. A., and Suh, G. S. (2013). Ionotropic glutamate receptors IR64a and IR8a form a functional odorant receptor complex in vivo in Drosophila. J. Neurosci. 33, 10741-10749. doi: 10.1523/JNEUROSCI.5419-12.2013

Ai, M., Min, S., Grosjean, Y., Leblanc, C., Bell, R., Benton, R., et al. (2010). Acid sensing by the Drosophila olfactory system. Nature 468, 691-695. doi: 10.1038/nature09537

Andersson, M. N., Keeling, C. I., and Mitchell, R. F. (2019). Genomic content of chemosensory genes correlates with host range in wood-boring beetles (Dendroctonus ponderosae, Agrilus planipennis, and Anoplophora glabripennis). BMC Genomics 20:690. doi: 10.1186/s12864-019-6054-X

Armstrong, N., Sun, Y., Chen, G. Q., and Gouaux, E. (1998). Structure of a glutamate-receptor ligand-binding core in complex with kainate. Nature 395, 913-917. doi: 10.1038/27692

Auer, T. O., Khallaf, M. A., Silbering, A. F., Zappia, G., Ellis, K., ÁlvarezOcaña, R., et al. (2020). Olfactory receptor and circuit evolution promote host specialization. Nature 579, 402-408. doi: 10.1038/s41586-020-2073-7

Balart-García, P., Cieslak, A., Escuer, P., Rozas, J., Ribera, I., and Fernández, R. (2020). Smelling in the dark: phylogenomic insights on the chemosensory system of a subterranean beetle. bioRxiv [Preprint]. doi: 10.1101/2020.10.22.350173

Bengtsson, J. M., Trona, F., Montagné, N., Anfora, G., Ignell, R., Witzgall, P., et al. (2012). Putative chemosensory receptors of the codling moth, Cydia pomonella, identified by antennal transcriptome analysis. PLoS ONE 7:e31620. doi: 10.1371/journal.pone.0031620

Benton, R., Vannice, K. S., Gomez-Diaz, C., and Vosshall, L. B. (2009). Variant ionotropic glutamate receptors as chemosensory receptors in Drosophila. Cell 136, 149-162. doi: 10.1016/j.cell.2008.12.001

Budelli, G., Ni, L., Berciu, C., van Giesen, L., Knecht, Z. A., Chang, E. C., et al. (2019). Ionotropic receptors specify the morphogenesis of phasic sensors controlling rapid thermal preference in Drosophila. Neuron 101, 738-747. doi: 10.1016/j.neuron.2018.12.022

Cao, D., Liu, Y., Wei, J., Liao, X., Walker, W. B., Li, J., et al. (2014). Identification of candidate olfactory genes in Chilo suppressalis by antennal transcriptome analysis. Int. J. Biol. Sci. 10, 846-860. doi: 10.7150/ijbs.9297

Carey, A. F., and Carlson, J. R. (2011). Insect olfaction from model systems to disease control. Proc. Natl. Acad. Sci. U.S.A. 108, 12987-12995. doi: $10.1073 /$ pnas. 1103472108

Chen, C., Buhl, E., Xu, M., Croset, V., Rees, J. S., Lilley, K. S., et al. (2015). Drosophila Ionotropic Receptor 25a mediates circadian clock resetting by temperature. Nature 527, 516-520. doi: 10.1038/nature16148

Chen, H. L., Stern, U., and Yang, C. H. (2019). Molecular control limiting sensitivity of sweet taste neurons in Drosophila. Proc. Natl. Acad. Sci. U.S.A. 116, 20158-20168. doi: 10.1073/pnas.1911583116

\section{SUPPLEMENTARY MATERIAL}

The Supplementary Material for this article can be found online at: https://www.frontiersin.org/articles/10.3389/fnmol. 2020.638839/full\#supplementary-material

Chen, Y., and Amrein, H. (2017). Ionotropic receptors mediate Drosophila oviposition preference through sour gustatory receptor neurons. Curr. Biol. 27, 2741-2750. doi: 10.1016/j.cub.2017.08.003

Chen, Y. D., and Dahanukar, A. (2017). Molecular and cellular organization of taste neurons in adult Drosophila pharynx. Cell Rep. 21, 2978-2991. doi: 10.1016/j.celrep.2017.11.041

Chen, Y. D., Park, S. J., Joseph, R. M., Ja, W. W., and Dahanukar, A. A. (2019). Combinatorial pharyngeal taste coding for feeding avoidance in adult Drosophila. Cell Rep. 29, 961-973. doi: 10.1016/j.celrep.2019.09.036

Chown, S. L., Sørensen, J. G., and Terblanche, J. S. (2011). Water loss in insects: an environmental change perspective. J. Insect Physiol. 57, 1070-1084. doi: 10.1016/j.jinsphys.2011.05.004

Cicconardi, F., Di Marino, D., Olimpieri, P. P., Arthofer, W., Schlick-Steiner, B. C., and Steiner, F. M. (2017). Chemosensory adaptations of the mountain fly Drosophila nigrosparsa (Insecta: Diptera) through genomics' and structural biology's lenses. Sci. Rep. 7:43770. doi: 10.1038/srep43770

Croset, V., Rytz, R., Cummins, S. F., Budd, A., Brawand, D., Kaessmann, H., et al. (2010). Ancient protostome origin of chemosensory ionotropic glutamate receptors and the evolution of insect taste and olfaction. PLoS Genet. 6:e1001064. doi: 10.1371/journal.pgen.1001064

Croset, V., Schleyer, M., Arguello, J. R., Gerber, B., and Benton, R. (2016). A molecular and neuronal basis for amino acid sensing in the Drosophila larva. Sci. Rep. 6:34871. doi: 10.1038/srep34871

de Bruyne, M., Foster, K., and Carlson, J. R. (2001). Odor coding in the Drosophila antenna. Neuron 30, 537-552. doi: 10.1016/S0896-6273(01)00289-6

Dippel, S., Kollmann, M., Oberhofer, G., Montino, A., Knoll, C., Krala, M., et al. (2016). Morphological and transcriptomic analysis of a beetle chemosensory system reveals a gnathal olfactory center. BMC Biol. 14:90. doi: 10.1186/s12915-016-0304-z

Enjin, A., Zaharieva, E. E., Frank, D. D., Mansourian, S., Suh, G. S., Gallio, M., et al. (2016). Humidity sensing in Drosophila. Curr. Biol. 26, 1352-1358. doi: 10.1016/j.cub.2016.03.049

Foelix, R. F., Stocker, R. F., and Steinbrecht, R. A. (1989). Fine structure of a sensory organ in the arista of Drosophila melanogaster and some other dipterans. Cell Tissue Res. 258, 277-287. doi: 10.1007/BF00239448

Frank, D. D., Enjin, A., Jouandet, G. C., Zaharieva, E. E., Para, A., Stensmyr, M. C., et al. (2017). Early integration of temperature and humidity stimuli in the Drosophila brain. Curr. Biol. 27, 2381-2388. doi: 10.1016/j.cub.2017.06.077

Ganguly, A., Pang, L., Duong, V. K., Lee, A., Schoniger, H., Varady, E., et al. (2017). A molecular and cellular context-dependent role for Ir76b in detection of amino acid taste. Cell Rep. 18, 737-750. doi: 10.1016/j.celrep.2016.12.071

Gao, X. J., Clandinin, T. R., and Luo, L. (2015). Extremely sparse olfactory inputs are sufficient to mediate innate aversion in Drosophila. PLoS ONE 10:e0125986. doi: 10.1371/journal.pone.0125986

Garrity, P. A., Goodman, M. B., Samuel, A. D., and Sengupta, P. (2010). Running hot and cold: behavioral strategies, neural circuits, and the molecular machinery for thermotaxis in C. elegans and Drosophila. Genes Dev. 24, 2365-2382. doi: 10.1101/gad.1953710

Glaser, N., Gallot, A., Legeai, F., Montagné, N., Poivet, E., Harry, M., et al. (2013). Candidate chemosensory genes in the Stemborer Sesamia nonagrioides. Int. J. Biol. Sci. 9, 481-495. doi: 10.7150/ijbs.6109

Gorter, J. A., Jagadeesh, S., Gahr, C., Boonekamp, J. J., Levine, J. D., and Billeter, J. C. (2016). The nutritional and hedonic value of food modulate sexual receptivity in Drosophila melanogaster females. Sci. Rep. 6:19441. doi: 10.1038/srep19441

Greppi, C., Laursen, W. J., Budelli, G., Chang, E. C., Daniels, A. M., van Giesen, L., et al. (2020). Mosquito heat seeking is driven by an ancestral cooling receptor. Science 367, 681-684. doi: 10.1126/science.aay9847 
Groh-Lunow, K. C., Getahun, M. N., Grosse-Wilde, E., and Hansson, B. S. (2014). Expression of ionotropic receptors in terrestrial hermit crab's olfactory sensory neurons. Front. Cell. Neurosci. 8:448. doi: 10.3389/fncel.2014.00448

Grosjean, Y., Rytz, R., Farine, J. P., Abuin, L., Cortot, J., Jefferis, G. S., et al. (2011). An olfactory receptor for food-derived odours promotes male courtship in Drosophila. Nature 478, 236-240. doi: 10.1038/nature10428

Hallem, E. A., Ho, M. G., and Carlson, J. R. (2004). The molecular basis of odor coding in the Drosophila antenna. Cell 117, 965-979. doi: 10.1016/j.cell.2004.05.012

Harrison, M. C., Jongepier, E., Robertson, H. M., Arning, N., Bitard-Feildel, T., Chao, H., et al. (2018). Hemimetabolous genomes reveal molecular basis of termite eusociality. Nat. Ecol. Evol. 2, 557-566. doi: 10.1038/s41559-017-0459-1

He, Z., Luo, Y., Shang, X., Sun, J. S., and Carlson, J. R. (2019). Chemosensory sensilla of the Drosophila wing express a candidate ionotropic pheromone receptor. PLoS Biol. 17:e2006619. doi: 10.1371/journal.pbio.2006619

Hueston, C. E., Olsen, D., Li, Q., Okuwa, S., Peng, B., Wu, J., et al. (2016). Chromatin modulatory proteins and olfactory receptor signaling in the refinement and maintenance of fruitless expression in olfactory receptor neurons. PLoS Biol. 14:e1002443. doi: 10.1371/journal.pbio.1002443

Hume, R. I., Dingledine, R., and Heinemann, S. F. (1991). Identification of a site in glutamate receptor subunits that controls calcium permeability. Science 253, 1028-1031. doi: 10.1126/science. 1653450

Hussain, A., Zhang, M., Ucpunar, H. K., Svensson, T., Quillery, E., Gompel, N., et al. (2016). Ionotropic chemosensory receptors mediate the taste and smell of polyamines. PLoS Biol. 14:e1002454. doi: 10.1371/journal.pbio.1002454

Jaeger, A. H., Stanley, M., Weiss, Z. F., Musso, P. Y., Chan, R. C., Zhang, H., et al. (2018). A complex peripheral code for salt taste in Drosophila. Elife 7:e37167. doi: 10.7554/eLife.37167.028

Jiang, L., Cheng, Y., Gao, S., Zhong, Y., Ma, C., Wang, T., et al. (2020). Emergence of social cluster by collective pairwise encounters in Drosophila. Elife 9:e51921. doi: 10.7554/eLife.51921

Jin, R., Singh, S. K., Gu, S., Furukawa, H., Sobolevsky, A. I., Zhou, J., et al. (2009). Crystal structure and association behaviour of the GluR2 amino-terminal domain. EMBO J 28, 1812-1823. doi: 10.1038/emboj.2009.140

Jones, W. D., Cayirlioglu, P., Kadow, I. G., and Vosshall, L. B. (2007). Two chemosensory receptors together mediate carbon dioxide detection in Drosophila. Nature 445, 86-90. doi: 10.1038/nature05466

Joseph, R. M., Sun, J. S., Tam, E., and Carlson, J. R. (2017). A receptor and neuron that activate a circuit limiting sucrose consumption. Elife 6:e24992. doi: 10.7554/eLife.24992.018

Jové, V., Gong, Z., Hol, F. J. H., Zhao, Z., Sorrells, T. R., Carroll, T. S., et al. (2020). Sensory discrimination of blood and floral nectar by Aedes aegypti mosquitoes. Neuron 108, 1163-1180. doi: 10.1016/j.neuron.2020.09.019

Knecht, Z. A., Silbering, A. F., Cruz, J., Yang, L., Croset, V., Benton, R., et al. (2017). Ionotropic Receptor-dependent moist and dry cells control hygrosensation in Drosophila. Elife 6:e26654. doi: 10.7554/eLife.26654.013

Knecht, Z. A., Silbering, A. F., Ni, L., Klein, M., Budelli, G., Bell, R., et al. (2016). Distinct combinations of variant ionotropic glutamate receptors mediate thermosensation and hygrosensation in Drosophila. Elife 5:e17879. doi: 10.7554/eLife.17879.011

Koh, T. W., He, Z., Gorur-Shandilya, S., Menuz, K., Larter, N. K., Stewart, S., et al. (2014). The Drosophila IR20a clade of ionotropic receptors are candidate taste and pheromone receptors. Neuron 83, 850-865. doi: 10.1016/j.neuron.2014.07.012

Kozma, M. T., Schmidt, M., Ngo-Vu, H., Sparks, S. D., Senatore, A., and Derby, C. D. (2018). Chemoreceptor proteins in the Caribbean spiny lobster, Panulirus argus: expression of ionotropic receptors, gustatory receptors, and TRP channels in two chemosensory organs and brain. PLoS ONE 13:e0203935. doi: 10.1371 /journal.pone.0203935

Kuner, T., Seeburg, P. H., and Guy, H. R. (2003). A common architecture for $\mathrm{K}+$ channels and ionotropic glutamate receptors? Trends Neurosci. 26, 27-32. doi: 10.1016/S0166-2236(02)00010-3

Latorre-Estivalis, J. M., Robertson, H. M., Walden, K. K., Ruiz, J., Gonçalves, L. O., Guarneri, A. A., et al. (2017). The molecular sensory machinery of a Chagas disease vector: expression changes through imaginal moult and sexually dimorphic features. Sci. Rep. 7:40049. doi: 10.1038/srep40049

Lee, Y., Poudel, S., Kim, Y., Thakur, D., and Montell, C. (2018). Calcium taste avoidance in Drosophila. Neuron 97, 67-74. doi: 10.1016/j.neuron.2017.11.038
Lei, J., Liu, Q., and Kadowaki, T. (2019). Honey bee parasitic mite contains the sensilla-rich sensory organ on the foreleg tarsus expressing ionotropic receptors with conserved functions. Front. Physiol. 10:556. doi: 10.3389/fphys.2019. 00556

Li, J., Chen, Q., Man, Y., Pei, D., and Wu, W. (2019). Variant ionotropic receptors are expressed in the antennae of Anopheles sinensis (Diptera: Culicidae). Biochem. Genet. 57, 571-582. doi: 10.1007/s10528-01909910-8

Liu, N. Y., Xu, W., Dong, S. L., Zhu, J. Y., Xu, Y. X., and Anderson, A. (2018). Genome-wide analysis of ionotropic receptor gene repertoire in Lepidoptera with an emphasis on its functions of Helicoverpa armigera. Insect Biochem. Mol. Biol. 99, 37-53. doi: 10.1016/j.ibmb.2018.05.005

Liu, N. Y., Xu, W., Papanicolaou, A., Dong, S. L., and Anderson, A. (2014). Identification and characterization of three chemosensory receptor families in the cotton bollworm Helicoverpa armigera. BMC Genomics 15:597. doi: 10.1186/1471-2164-15-597

Liu, Z., Wang, X., Lei, C., and Zhu, F. (2017). Sensory genes identification with head transcriptome of the migratory armyworm, Mythimna separata. Sci. Rep. 7:46033. doi: 10.1038/srep46033

Ma, C., Zhao, C., Cui, S., Zhang, Y., Chen, G., Chen, H., et al. (2019). Identification of candidate chemosensory genes of Ophraella communa LeSage (Coleoptera: Chrysomelidae) based on antennal transcriptome analysis. Sci. Rep. 9:15551. doi: 10.1038/s41598-019-52149-x

Macharia, R., Mireji, P., Murungi, E., Murilla, G., Christoffels, A., Aksoy, S., et al. (2016). Genome-wide comparative analysis of chemosensory gene families in five tsetse fly species. PLoS Negl. Trop. Dis. 10:e0004421. doi: 10.1371/journal.pntd.0004421

Manoli, D. S., Foss, M., Villella, A., Taylor, B. J., Hall, J. C., and Baker, B. S. (2005). Male-specific fruitless specifies the neural substrates of Drosophila courtship behaviour. Nature 436, 395-400. doi: 10.1038/nature03859

Marin, E. C., Büld, L., Theiss, M., Sarkissian, T., Roberts, R. J. V., Turnbull, R., et al. (2020). Connectomics analysis reveals first-, second-, and third-order thermosensory and hygrosensory neurons in the adult Drosophila brain. Curr. Biol. 30, 3167-3182. doi: 10.1016/j.cub.2020.06.028

Masse, N. Y., Turner, G. C., and Jefferis, G. S. (2009). Olfactory information processing in Drosophila. Curr. Biol. 19, R700-R713. doi: 10.1016/j.cub.2009.06.026

Matthews, B. J., Dudchenko, O., Kingan, S. B., Koren, S., Antoshechkin, I., Crawford, J. E., et al. (2018). Improved reference genome of Aedes aegypti informs arbovirus vector control. Nature 563, 501-507. doi: $10.1038 / \mathrm{s} 41586-018-0692-\mathrm{z}$

Mayer, M. L. (2006). Glutamate receptors at atomic resolution. Nature 440, 456-462. doi: 10.1038/nature04709

Mayer, M. L., and Armstrong, N. (2004). Structure and function of glutamate receptor ion channels. Annu. Rev. Physiol. 66, 161-181. doi: 10.1146/annurev.physiol.66.050802.084104

Menuz, K., Larter, N. K., Park, J., and Carlson, J. R. (2014). An RNA-seq screen of the Drosophila antenna identifies a transporter necessary for ammonia detection. PLoS Genet. 10:e1004810. doi: 10.1371/journal.pgen.10 04810

Min, S., Ai, M., Shin, S. A., and Suh, G. S. (2013). Dedicated olfactory neurons mediating attraction behavior to ammonia and amines in Drosophila. Proc. Natl. Acad. Sci. U.S.A. 110, E1321-E1329. doi: 10.1073/pnas.1215680110

Missbach, C., Dweck, H. K., Vogel, H., Vilcinskas, A., Stensmyr, M. C., Hansson, B. S., et al. (2014). Evolution of insect olfactory receptors. Elife 3:e02115. doi: $10.7554 /$ eLife.02115.030

Montell, C. (2009). A taste of the Drosophila gustatory receptors. Curr. Opin. Neurobiol. 19, 345-353. doi: 10.1016/j.conb.2009.07.001

Ng, R., Salem, S. S., Wu, S. T., Wu, M., Lin, H. H., Shepherd, A. K., et al. (2019). Amplification of Drosophila olfactory responses by a DEG/ENaC channel. Neuron 104, 947-959. doi: 10.1016/j.neuron.2019.08.041

Ni, L., Klein, M., Svec, K. V., Budelli, G., Chang, E. C., Ferrer, A. J., et al. (2016). The ionotropic receptors IR21a and IR25a mediate cool sensing in Drosophila. Elife 5:e13254. doi: 10.7554/eLife.13254.015

Poivet, E., Gallot, A., Montagné, N., Glaser, N., Legeai, F., and JacquinJoly, E. (2013). A comparison of the olfactory gene repertoires of adults and larvae in the noctuid moth Spodoptera littoralis. PLoS ONE 8:e60263. doi: 10.1371 /journal.pone. 0060263 
Prieto-Godino, L. L., Rytz, R., Bargeton, B., Abuin, L., Arguello, J. R., Peraro, M. D., et al. (2016). Olfactory receptor pseudo-pseudogenes. Nature 539, 93-97. doi: $10.1038 /$ nature19824

Prieto-Godino, L. L., Rytz, R., Cruchet, S., Bargeton, B., Abuin, L., Silbering, A. F., et al. (2017). Evolution of acid-sensing olfactory circuits in Drosophilids. Neuron 93, 661-676. doi: 10.1016/j.neuron.2016.12.024

Raji, J. I., Melo, N., Castillo, J. S., Gonzalez, S., Saldana, V., Stensmyr, M. C., et al. (2019). Aedes aegypti mosquitoes detect acidic volatiles found in human odor using the IR8a pathway. Curr. Biol. 29, 1253-1262. doi: 10.1016/j.cub.2019.02.045

Rimal, S., and Lee, Y. (2018). The multidimensional ionotropic receptors of Drosophila melanogaster. Insect Mol. Biol. 27, 1-7. doi: 10.1111/imb.12347

Rimal, S., Sang, J., Poudel, S., Thakur, D., Montell, C., and Lee, Y. (2019). Mechanism of acetic acid gustatory repulsion in Drosophila. Cell Rep. 26, 1432-1442. doi: 10.1016/j.celrep.2019.01.042

Rist, A., and Thum, A. S. (2017). A map of sensilla and neurons in the taste system of Drosophila larvae. J. Comp. Neurol. 525, 3865-3889. doi: 10.1002/cne.24308

Robertson, H. M., Baits, R. L., Walden, K. K. O., Wada-Katsumata, A., and Schal, C. (2018). Enormous expansion of the chemosensory gene repertoire in the omnivorous German cockroach Blattella germanica. J. Exp. Zool. B Mol. Dev. Evol. 330, 265-278. doi: 10.1002/jez.b.22797

Robertson, H. M., Robertson, E. C. N., Walden, K. K. O., Enders, L. S., and Miller, N. J. (2019). The chemoreceptors and odorant binding proteins of the soybean and pea aphids. Insect Biochem. Mol. Biol. 105, 69-78. doi: 10.1016/j.ibmb.2019.01.005

Rojas, V., Jiménez, H., Palma-Millanao, R., González-González, A., Machuca, J., Godoy, R., et al. (2018). Analysis of the grapevine moth Lobesia botrana antennal transcriptome and expression of odorant-binding and chemosensory proteins. Comp. Biochem. Physiol. Part D Genomics Proteomics 27, 1-12. doi: 10.1016/j.cbd.2018.04.003

Rytz, R., Croset, V., and Benton, R. (2013). Ionotropic receptors (IRs): chemosensory ionotropic glutamate receptors in Drosophila and beyond. Insect Biochem. Mol. Biol. 43, 888-897. doi: 10.1016/j.ibmb.2013.02.007

Sanchez-Alcaniz, J. A., Silbering, A. F., Croset, V., Zappia, G., Sivasubramaniam, A. K., Abuin, L., et al. (2018). An expression atlas of variant ionotropic glutamate receptors identifies a molecular basis of carbonation sensing. Nat. Commun. 9:4252. doi: 10.1038/s41467-018-06453-1

Senthilan, P. R., Piepenbrock, D., Ovezmyradov, G., Nadrowski, B., Bechstedt, S., Pauls, S., et al. (2012). Drosophila auditory organ genes and genetic hearing defects. Cell 150, 1042-1054. doi: 10.1016/j.cell.2012.06.043

Shanbhag, S. R., Singh, K., and Singh, R. N. (1995). Fine structure and primary sensory projections of sensilla located in the sacculus of the antenna of Drosophila melanogaster. Cell Tissue Res. 282, 237-249. doi: $10.1007 / \mathrm{BF} 00319115$

Silbering, A. F., Rytz, R., Grosjean, Y., Abuin, L., Ramdya, P., Jefferis, G. S., et al. (2011). Complementary function and integrated wiring of the evolutionarily distinct Drosophila olfactory subsystems. J. Neurosci. 31, 13357-13375. doi: 10.1523/JNEUROSCI.2360-11.2011

Sobolevsky, A. I., Rosconi, M. P., and Gouaux, E. (2009). X-ray structure, symmetry, and mechanism of an AMPA-subtype glutamate receptor. Nature 462, 745-756. doi: 10.1038/nature08624

Squire, L. R., Bloom, F. E., McConnell, S. K., Roberts, J. L., Spitzer, N. C., and Zigmond, M. J. (2003). Fundamental Neuroscience. Cambridge, MA: Academic Press.

Steck, K., Walker, S. J., Itskov, P. M., Baltazar, C., Moreira, J. M., and Ribeiro, C. (2018). Internal amino acid state modulates yeast taste neurons to support protein homeostasis in Drosophila. Elife 7:e31625. doi: 10.7554/eLife.31625

Stewart, S., Koh, T. W., Ghosh, A. C., and Carlson, J. R. (2015). Candidate ionotropic taste receptors in the Drosophila larva. Proc. Natl. Acad. Sci. U.S.A. 112, 4195-4201. doi: 10.1073/pnas.1503292112

Stockinger, P., Kvitsiani, D., Rotkopf, S., Tirián, L., and Dickson, B. J. (2005). Neural circuitry that governs Drosophila male courtship behavior. Cell 121, 795-807. doi: 10.1016/j.cell.2005.04.026

Sun, Y. L., Dong, J. F., Gu, N., and Wang, S. L. (2020). Identification of candidate chemosensory receptors in the antennae of the variegated cutworm, Peridroma saucia Hübner, based on a transcriptome analysis. Front. Physiol. 11:39. doi: $10.3389 /$ fphys.2020.00039
Tauber, J. M., Brown, E. B., Li, Y., Yurgel, M. E., Masek, P., and Keene, A. C. (2017). A subset of sweet-sensing neurons identified by IR56d are necessary and sufficient for fatty acid taste. PLoS Genet. 13:e1007059. doi: 10.1371/journal.pgen.1007059

van der Goes van Naters, W., and Carlson, J. R. (2007). Receptors and neurons for fly odors in Drosophila. Curr. Biol. 17, 606-612. doi: 10.1016/j.cub.2007.02.043

van Giesen, L., and Garrity, P. A. (2017). More than meets the IR: the expanding roles of variant ionotropic glutamate receptors in sensing odor, taste, temperature, and moisture. F1000Res 6:1753. doi: 10.12688/f1000research.12013.1

van Giesen, L., Hernandez-Nunez, L., Delasoie-Baranek, S., Colombo, M., Renaud, P., Bruggmann, R., et al. (2016). Multimodal stimulus coding by a gustatory sensory neuron in Drosophila larvae. Nat. Commun. 7:10687. doi: $10.1038 /$ ncomms 10687

van Schooten, B., Jiggins, C. D., Briscoe, A. D., and Papa, R. (2016). Genomewide analysis of ionotropic receptors provides insight into their evolution in Heliconius butterflies. BMC Genomics 17:254. doi: 10.1186/s12864-016-2572-y

Vosshall, L. B., and Stocker, R. F. (2007). Molecular architecture of smell and taste in Drosophila. Annu. Rev. Neurosci. 30, 505-533. doi: 10.1146/annurev.neuro.30.051606.094306

Vosshall, L. B., Wong, A. M., and Axel, R. (2000). An olfactory sensory map in the fly brain. Cell 102, 147-159. doi: 10.1016/S0092-8674(00)00021-0

Wu, Y. M., Liu, Y. Y., and Chen, X. S. (2020). Genomic content of chemosensory receptors in two sister blister beetles facilitates characterization of chemosensory evolution. BMC Genomics 21:589. doi: 10.1186/s12864-020-06974-4

Xu, J. W., Zhu, X. Y., Chao, Q. J., Zhang, Y. J., Yang, Y. X., Wang, R. R., et al. (2019). Chemosensory gene families in the oligophagous pear pest Cacopsylla chinensis (Hemiptera: Psyllidae). Insects 10:175. doi: 10.3390/insects10060175

Yanagawa, A., Couto, A., Sandoz, J. C., Hata, T., Mitra, A., Ali Agha, M., et al. (2019). LPS perception through taste-induced reflex in Drosophila melanogaster. J. Insect Physiol. 112, 39-47. doi: 10.1016/j.jinsphys.2018. 12.001

Yang, H., Dong, J., Sun, Y. L., Hu, Z., Lyu, Q. H., and Li, D. (2020). Identification and expression profiles of candidate chemosensory receptors in Histia rhodope (Lepidoptera: Zygaenidae). PeerJ 8:e10035. doi: 10.7717/peerj.10035

Yang, S., Cao, D., Wang, G., and Liu, Y. (2017). Identification of genes involved in chemoreception in Plutella xyllostella by antennal transcriptome analysis. Sci. Rep. 7:11941. doi: 10.1038/s41598-017-11646-7

Yao, C. A., Ignell, R., and Carlson, J. R. (2005). Chemosensory coding by neurons in the coeloconic sensilla of the Drosophila antenna. J. Neurosci. 25, 8359-8367. doi: 10.1523/JNEUROSCI.2432-05.2005

Yin, N. N., Nuo, S. M., Xiao, H. Y., Zhao, Y. J., Zhu, J. Y., and Liu, N. Y. (2020). The ionotropic receptor gene family in Lepidoptera and Trichoptera: annotation, evolutionary, and functional perspectives. Genomics (in press). doi: 10.1016/j.ygeno.2020.09.056

Zbinden, M., Berthod, C., Montagné, N., Machon, J., Léger, N., Chertemps, T., et al. (2017). Comparative study of chemosensory organs of shrimp from hydrothermal vent and coastal environments. Chem. Senses 42, 319-331. doi: 10.1093/chemse/bjx007

Zhang, Y. V., Ni, J., and Montell, C. (2013). The molecular basis for attractive salt-taste coding in Drosophila. Science 340, 1334-1338. doi: 10.1126/science.1234133

Zuo, J., De Jager, P. L., Takahashi, K. A., Jiang, W., Linden, D. J., and Heintz, N. (1997). Neurodegeneration in Lurcher mice caused by mutation in delta2 glutamate receptor gene. Nature 388, 769-773. doi: 10.1038/42009

Conflict of Interest: The author declares that the research was conducted in the absence of any commercial or financial relationships that could be constructed as a potential conflict of interest.

Copyright (c) $2021 \mathrm{Ni}$. This is an open-access article distributed under the terms of the Creative Commons Attribution License (CC BY). The use, distribution or reproduction in other forums is permitted, provided the original author $(s)$ and the copyright owner(s) are credited and that the original publication in this journal is cited, in accordance with accepted academic practice. No use, distribution or reproduction is permitted which does not comply with these terms. 